\title{
SECOND-ORDER PROPERTIES AND CENTRAL LIMIT THEORY FOR THE VERTEX PROCESS OF ITERATION INFINITELY DIVISIBLE AND ITERATION STABLE RANDOM TESSELLATIONS IN THE PLANE
}

\author{
TOMASZ SCHREIBER, ${ }^{*}$ Nicolaus Copernicus University, Toruń \\ CHRISTOPH THÄLE, ${ }^{* *}$ University of Fribourg
}

\begin{abstract}
The point process of vertices of an iteration infinitely divisible or, more specifically, of an iteration stable random tessellation in the Euclidean plane is considered. We explicitly determine its covariance measure and its pair-correlation function, as well as the crosscovariance measure and the cross-correlation function of the vertex point process and the random length measure in the general nonstationary regime. We also give special formulae in the stationary and isotropic setting. Exact formulae are given for vertex count variances in compact and convex sampling windows, and asymptotic relations are derived. Our results are then compared with those for a Poisson line tessellation having the same length density parameter. Moreover, a functional central limit theorem for the joint process of suitably rescaled total edge counts and edge lengths is established with the process $(\xi, t \xi), t>0$, arising in the limit, where $\xi$ is a centered Gaussian variable with explicitly known variance.

Keywords: Central limit theorem; covariance measure; cross correlation; Markov process; iteration/nesting; martingale; pair-correlation function; random tessellation; stochastic stability; stochastic geometry

2010 Mathematics Subject Classification: Primary 60D05
\end{abstract}

Secondary 60G55; 60J75; 60F05

\section{Introduction}

Random tessellations have attracted particular interest in stochastic geometry because of their wide applications ranging from classical geological problems to recent developments in telecommunication; see [10] and [15]. It is one of the main purposes of the related theory to develop new classes of random tessellations that are mathematically tractable and yet rich enough in structure so that they may serve as new reference models for applications beside the classical Poisson hyperplane and the Poisson-Voronoi tessellation. A very recent model, the so-called random STIT (stable under iteration) tessellations, was introduced by Nagel and Weiss in [8]. One of the main features of these tessellations that distinguishes them from the abovementioned model classes is the property that their cells are not side-to-side; see Figure 1. This causes new geometric effects whose planar first-order properties in terms of mean values were explored in [9]. This paper will deal with second-order characteristics and central limit theory for the planar case, a topic that was considered first by Weiss et al. in [16] and later studied

Received 11 May 2010; revision received 28 August 2010.

* Postal address: Faculty of Mathematics and Computer Science, Nicolaus Copernicus University, Ulica Chopina 12/18, 87-100 Toruń, Poland. Email address: tomeks@mat.umk.pl

** Current address: Department of Mathematics, University of Osnabrück, D-49076 Osnabrück, Germany.

Email address: christoph.thaele@uni-osnabrueck.de 

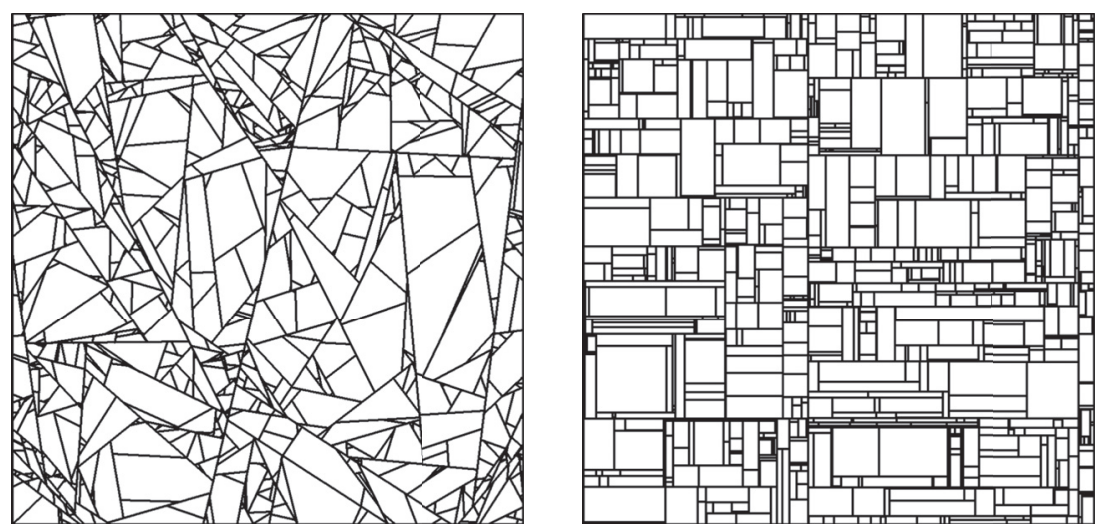

FIGURE 1: Realizations of two STIT tessellations with different driving measures, the invariant measure (left) and a measure concentrated on lines pointing in only two orthogonal directions with weight $\frac{1}{2}$ (right).

in [12], where, beside other characteristics, the variance of the total edge length was determined. Here, in contrast, we will deal with second-order properties of the point process of vertices of the tessellation. To provide more general results, we will not restrict our attention to the class of stationary random iteration stable tessellations (STIT tessellations), but instead we will study the larger class of nonstationary random iteration infinitely divisible tessellations in the plane. They were introduced in [12] as generalizations of STIT tessellations and in Subsection 2.1 we will recall their construction in the spirit of Mecke-Nagel-Weiss (MNW) [8]. It is an important observation that this spatio-temporal MNW construction can be interpreted as a continuous-time Markov process on the space of tessellations, whence the general theory of Markov processes is available. Before extending some mean value relations from the stationary iteration stable to the nonstationary iteration infinitely divisible case in Subsection 2.3, we will formulate the main technical tools from the theory of Markov processes in Subsection 2.2, on which our main results are based. They are the content of Sections 3 and 4 . The variance of the total number of vertices and that of the total number of maximal edges in a bounded observation window will be calculated in Subsection 3.1 for very general driving measures, whereas in Subsection 3.4 we specialize to the motion-invariant case. The vertex pair-correlation measure for general line measures is considered in Subsection 3.2 and the considerations are specialized again to the stationary and isotropic setting in Subsection 3.5. Moreover, we determine in Subsection 3.3 the exact cross-covariance measure of the vertex point process and the length measure in the general case and provide specialized formulae in Subsection 3.6 for the stationary and isotropic regime. Here, the most explicit formulae are available. Another topic treated there concerns the variance asymptotics for a sequence $W_{R}$ of growing windows as $R$ tends to $\infty$. Based on these results, in Section 4 we deal with the central limit problem. It will be shown that a certain rescaled bivariate process of edge counts and edge lengths of a STIT tessellation converges to the process $(\xi, t \xi)$, where $\xi$ is a centered normal random variable with an explicitly known variance.

We would like to emphasize that our results may be of interest in the context of statistical model fitting of random tessellations to real data; see [10]. Our central limit theory could be a base for statistical inference of tessellation models and related functionals, and asymptotic confidence intervals and statistical tests can be derived from them, since we make the first- and second-order moments explicitly available. 
For general notions and notation related to stochastic geometry and the theory of random tessellations, which are used in this paper, we refer the reader to [11] and [15].

\section{Background material}

\subsection{Iteration infinitely divisible and iteration stable random tessellations}

Consider a compact and convex set $W \subset \mathbb{R}^{2}$ with nonempty interior and a diffuse (nonatomic) measure $\Lambda$ on the space of $\left[\mathbb{R}^{2}\right]$ of lines in the plane enjoying the local finiteness property, stating that $\Lambda([C])<\infty$ for any compact $C \subset \mathbb{R}^{2}$, under the usual notation

$$
[C]=\{\text { lines } L: L \cap C \neq \varnothing\},
$$

letting $[C]$ stand for the set of lines that have nonempty intersection with $C$. Below we briefly describe the construction of an iteration infinitely divisible tessellation in $W$, the construction is called the MNW construction to honor its inventors, Mecke, Nagel, and Weiss, who introduced it in [8]. To begin, assign to $W$ an exponentially distributed random lifetime with parameter given by $\Lambda([W])$. Upon expiry of this random time, the cell $W$ dies and splits into two subcells, $W^{+}$and $W^{-}$, separated by a line in $[W]$ chosen according to the law $\Lambda([W])^{-1} \Lambda(\cdot \cap[W])$. The resulting new cells $W^{+}$and $W^{-}$are again assigned independent exponential lifetimes with respective parameters $\Lambda\left(\left[W^{+}\right]\right)$and $\Lambda\left(\left[W^{-}\right]\right)$, whereupon the construction continues recursively and independently in each of the subcells $W^{+}$and $W^{-}$until some deterministic time threshold $t>0$ is reached. The random tessellation constructed in $W$ by time $t$ will be denoted by $Y(t, W)$. Stated formally, by $Y(t, W)$ we denote the random closed subset of $W$ arising as the union of the boundaries of cells constructed by time $t$. The cell-splitting edges are called the maximal edges (in the related literature they are often called I-segments, as they assume shapes similar to that of the literal I) of the tessellation $Y(t, W)$ and the family of all such edges is denoted by MaxEdges $(Y(t, W))$. Note that such maximal edges can be further subdivided between their birth time and time $t$, that is, in the course of the MNW dynamics there can appear additional vertices in their relative interiors. Thus, we have a distinction between maximal edges with a possible interior structure and those edges which are not maximal (for example, the sides of cells or the primitive elements that are bounded by vertices but have no interior structure).

In general, the random tessellations $Y(t, W)$ in $W$ do not have to arise as windowed restrictions of stationary (stochastically translation invariant) or isotropic (stochastically rotation invariant) processes. If we assume in addition though that the driving measure $\Lambda$ has, under polar parametrization, the product structure $\Lambda=\tau \ell_{+} \otimes \mathcal{R}$ with $\tau$ a positive constant, $\ell_{+}$ the Lebesgue measure on $\mathbb{R}_{+}$, and a spherical directional distribution $\mathcal{R}$ on the unit circle $\S_{1}$, then $Y(t, W)$ has the property of being a suitable windowed restriction of a stationary random field. The random tessellation $Y(t, W)$ is furthermore isotropic in a similar restrictionwise sense if and only if $\mathcal{R}$ is the uniform distribution, i.e. if and only if $\Lambda$ is a multiple of the standard motion-invariant measure $\Lambda_{\text {iso }}:=\ell_{+} \otimes v_{1}$ of unit length intensity on the space of lines in the plane, where $\nu_{1}$ is the uniform distribution on $\delta_{1}$.

We now review some of the important properties of the tessellation $Y(t, W)$, proofs of which can be found in [7], [8], and [12]. We formulate them only for the planar case, even if they are true in higher dimensions as well.

1. The random tessellations $Y(t, W)$ are consistent in $W$, that is, $Y(t, V) \cap W \stackrel{\mathrm{D}}{=} Y(t, W)$ for $W \subseteq V$ and $V, W$ compact convex, where ' $\stackrel{\mathrm{D}}{=}$ ' denotes equality in distribution. This 
implies the existence of the whole-plane tessellation $Y(t)$ such that $Y(t, W) \stackrel{\mathrm{D}}{=} Y(t) \cap W$ for each compact convex $W$.

2. The MNW construction satisfies the Markov property with respect to the iteration of tessellations in the time parameter $t$, i.e.

$$
Y(s, W) \boxplus Y(t, W) \stackrel{\mathrm{D}}{=} Y(s+t, W)
$$

where ' $\boxplus$ ' denotes the operation of iteration for whose exact definition we refer the reader to the abovementioned papers. This operation can roughly be explained as follows. Let $Y_{0}$ be a random tessellation, and let $T_{1}, T_{2}, \ldots$ be a sequence of independent and identically distributed random tessellations in the plane. To each cell $c_{k}$ of the frame tessellation $Y_{0}$ we associate the tessellation $T_{k}$. Now, we make a local superposition of $Y_{0}$ and the cutouts of $T_{k}$ in each cell $c_{k}$ of the frame tessellation. If we let $Y_{1}$ be a tessellation with the same distribution as $T_{k}$ for any $k=1,2, \ldots$, we denote the outcome of this procedure of local superposition by $Y_{0} \boxplus Y_{1}$ and call it the iteration of $Y_{0}$ with $Y_{1}$.

3. It directly follows from the above Markov property that the random tessellations $Y(t, W)$ are infinitely divisible with respect to iteration, i.e.

$$
Y(t, W) \stackrel{\mathrm{D}}{=} \underbrace{\left(\frac{t}{n}, W\right) \boxplus \cdots \boxplus Y\left(\frac{t}{n}, W\right)}_{n}
$$

for any $n \in \mathbb{N}$ and any compact convex $W$. For this reason, the tessellations $Y(t, W)$ are referred to as iteration infinitely divisible random tessellations. However, it is worth pointing out that it is currently not known whether any iteration infinitely divisible random tessellation can be obtained by the MNW construction. If, in addition, the driving measure $\Lambda$ is translation invariant, it can be verified that $Y(t, W)$ is even stable under iteration, i.e.

$$
Y(t) \stackrel{\mathrm{D}}{=} n(\underbrace{Y(t) \boxplus \cdots \boxplus Y(t)}_{n}), \quad n \in \mathbb{N},
$$

where $n(\cdot)$ denotes the dilation with a factor $n$, i.e. $n Y=\{n x: x \in Y\}$. Note that this equation must be understood symbolically, since the operation of iteration involves a sequence of independent and identically distributed random tessellations, but we adopt here the usual convention from the earlier work [7] and suppress writing out the whole sequence. Thus, in the stationary case we are in fact dealing with iteration stable random tessellations or STIT tessellations for short.

3. The intersection of an iteration infinitely divisible random tessellation $Y(t, W)$ having driving measure $\Lambda$ with an arbitrary line $L \in[W]$ is a Poisson point process with intensity measure $A \mapsto t \Lambda([A])$, where $A \subset W \cap L$ is a Borel set. In particular, for $x, y \in W$, the probability that $x$ and $y$ belong to the same cell of $Y(t, W)$ equals

$$
\mathrm{P}(x \text { and } y \text { are in the same cell of } Y(t, W))=\mathrm{e}^{-t \Lambda([\overline{x y}])},
$$

where $\overline{x y} \subset W$ denotes the line segment joining the points $x$ and $y$. 


\subsection{Martingales in the MNW construction}

As already mentioned in the introduction, the MNW construction of iteration infinitely divisible or iteration stable random tessellations $Y(t, W)$ in finite volumes $W \subset \mathbb{R}^{2}$ for general locally finite and diffuse driving measures $\Lambda$ satisfies the Markov property in the continuoustime parameter $t$. In our previous work [12] we used this fact combined with the classical theory of martingale problems for pure jump Markov processes to construct a class of natural martingales associated to the MNW process. In this paper we need only a part of that theory. To formulate the required results, for a bounded and measurable functional $\phi$ of a line segment (tessellation edge) and for a tessellation $Y$, usually taken to be a realization of $Y(t, W)$ for some $t>0$, write

$$
\Sigma_{\phi}(Y)=\sum_{e \in \operatorname{MaxEdges}(Y)} \phi(e) .
$$

Note that, with $Y$ as above, for each straight line $L \in[W]$, the intersection $Y \cap L$ is just a onedimensional tessellation of $L \cap W$ which can be identified with the collection $\operatorname{Segments}(Y \cap L)$ of its constituent segments. Bearing this in mind we write

$$
A_{\phi}(Y)=\int_{[W]} \sum_{e \in \operatorname{Segments}(Y \cap L \cap W)} \phi(e) \Lambda(\mathrm{d} L) .
$$

It is also convenient to introduce the bar notation for centered versions of these quantities with $Y=Y(t, W)$, that is,

$$
\bar{\Sigma}_{\phi}(Y(t, W)):=\Sigma_{\phi}(Y(t, W))-\mathrm{E} \Sigma_{\phi}(Y(t, W))
$$

and likewise

$$
\bar{A}_{\phi}(Y(t, W)):=A_{\phi}(Y(t, W))-\mathrm{E} A_{\phi}(Y(t, W)) .
$$

With this notation, in view of Equation (41) of [12] we have the following result.

Proposition 1. For bounded measurable segment functionals $\phi$ and $\psi$, the stochastic processes

$$
\Sigma_{\phi}(Y(t, W))-\int_{0}^{t} A_{\phi}(Y(s, W)) \mathrm{d} s
$$

and

$$
\begin{aligned}
& \bar{\Sigma}_{\phi}(Y(t, W)) \bar{\Sigma}_{\psi}(Y(t, W))-\int_{0}^{t} A_{\phi \psi}(Y(s, W)) \mathrm{d} s \\
& \quad-\int_{0}^{t}\left[\bar{A}_{\phi}(Y(s, W)) \bar{\Sigma}_{\psi}(Y(s, W))+\bar{A}_{\psi}(Y(s, W)) \bar{\Sigma}_{\phi}(Y(s, W))\right] \mathrm{d} s
\end{aligned}
$$

are martingales with respect to the filtration $\mathfrak{I}_{t}$ generated by $(Y(s, W))_{0 \leq s \leq t}$.

\subsection{Mean values for edge and vertex processes}

This subsection recapitulates some basic first-order properties of iteration infinitely divisible tessellations, mostly known in the stationary setup, for the sake of reference in further sections. Using (2) with $\phi \equiv 1$ yields, upon taking expectations,

$$
\mathrm{E} \Sigma_{1}(Y(t, W))=\int_{0}^{t} \mathrm{E} A_{1}(Y(s, W)) \mathrm{d} s .
$$


However, in view of definition (1) it is easily verified that

$$
A_{1}(Y)=\Lambda([W])+\sum_{e \in \operatorname{MaxEdges}(Y)} \Lambda([e])=\Lambda([W])+\Sigma_{\Lambda[\cdot]}(Y),
$$

where $\Lambda[\cdot]$ stands for the edge functional $e \mapsto \Lambda([e])$. Applying (2) once again with $\phi=\Lambda[\cdot]$ leads to

$$
\mathrm{E} \Sigma_{\Lambda[\cdot]}(Y(t, W))=\int_{0}^{t} \operatorname{E} A_{\Lambda[\cdot]}(Y(s, W)) \mathrm{d} s
$$

However,

$$
A_{\Lambda[\cdot]}(Y(s, W))=\int_{[W]} \Lambda([L \cap W]) \Lambda(\mathrm{d} L)=\langle\langle\Lambda \cap \Lambda\rangle\rangle(W) \quad \text { for all } s \in(0, t],
$$

where the locally finite point-intersection measure $\langle\langle\Lambda \cap \Lambda\rangle\rangle$ on $\mathbb{R}^{2}$ is given by

$$
\langle\langle\Lambda \cap \Lambda\rangle\rangle:=\int_{\left[\mathbb{R}^{2}\right]} \int_{[L]} \delta_{L \cap L^{\prime}} \Lambda\left(\mathrm{d} L^{\prime}\right) \Lambda(\mathrm{d} L),
$$

where, recall, $\delta_{(\cdot)}$ stands for the Dirac unit mass at the argument, so that, in other words,

$$
\langle\langle\Lambda \cap \Lambda\rangle\rangle(A)=(\Lambda \times \Lambda)\left\{\left(L_{1}, L_{2}\right) \in[A] \times[A], L_{1} \cap L_{2} \in A\right\}, \quad A \subseteq \mathbb{R}^{2} .
$$

Hence,

$$
\mathrm{E} \Sigma_{\Lambda[\cdot]}(Y(s, W))=s\langle\langle\Lambda \cap \Lambda\rangle\rangle(W) .
$$

Combining (4), (5), and (8) finally yields

$$
\mathrm{E} \Sigma_{1}(Y(t, W))=t \Lambda([W])+\frac{t^{2}}{2}\langle\langle\Lambda \cap \Lambda\rangle\rangle(W) .
$$

Note that (9), when specialized to the translation-invariant setup, contains an extra boundary correction term, $t \Lambda([W])$, in comparison to the classical mean value formula for the iteration stable (STIT) random tessellations given in [9], which says that the density of maximal edges in $W$ is just $t^{2}\langle\langle\Lambda \cap \Lambda\rangle\rangle(W) / 2$. This additional boundary correction term, $t \Lambda([W])$, comes from the fact that we count edges rather than edge midpoints. Thus, it can happen that in two neighboring regions we observe two distinct edges which may coalesce into one edge when putting these regions together into one area.

\section{Second-order theory for edge and vertex processes}

\subsection{Variance calculation for the general case}

We consider the most general case first and study iteration infinitely divisible random tessellations $Y(t, W)$ with general locally finite and nonatomic driving measures $\Lambda$. We fix $t>0$ and a compact and convex observation window $W \subset \mathbb{R}^{2}$ as in Subsection 2.1. First we use (3) with $\phi=\psi \equiv 1$ to conclude that

$$
\bar{\Sigma}_{1}^{2}(Y(t, W))-\int_{0}^{t} A_{1}(Y(s, W)) \mathrm{d} s-2 \int_{0}^{t} \bar{A}_{1}(Y(s, W)) \bar{\Sigma}_{1}(Y(s, W)) \mathrm{d} s
$$


is a martingale with respect to $\mathfrak{I}_{t}$. Using (5) and taking expectations, we obtain

$$
\begin{aligned}
\operatorname{var}\left(\Sigma_{1}(Y(t, W))\right)= & t \Lambda([W])+\int_{0}^{t} \mathrm{E} \Sigma_{\Lambda[\cdot]}(Y(s, W)) \mathrm{d} s \\
& +2 \int_{0}^{t} \operatorname{cov}\left(\Sigma_{\Lambda[\cdot]}(Y(s, W)), \Sigma_{1}(Y(s, W))\right) \mathrm{d} s .
\end{aligned}
$$

It remains to find an expression for the covariance $\operatorname{cov}\left(\Sigma_{\Lambda[\cdot]}(Y(s, W)), \Sigma_{1}(Y(s, W))\right)$. Such an expression can be found by applying (3) once again, this time with $\phi=1$ and $\psi=\Lambda[\cdot]$, and $t$ replaced by $s$ and $s$ replaced by $u$. In this way we obtain

$$
\begin{aligned}
\operatorname{cov}\left(\Sigma_{\Lambda[\cdot]}(Y(s, W)), \Sigma_{1}(Y(s, W))\right)= & \int_{0}^{s} \operatorname{E} A_{\Lambda[\cdot]}(Y(u, W)) \mathrm{d} u \\
& +\int_{0}^{s} \operatorname{cov}\left(A_{1}(Y(u, W)), \Sigma_{\Lambda[\cdot]}(Y(u, W))\right) \mathrm{d} u \\
& +\int_{0}^{s} \operatorname{cov}\left(A_{\Lambda[\cdot]}(Y(u, W)), \Sigma_{1}(Y(u, W))\right) \mathrm{d} u .
\end{aligned}
$$

In view of (6), $A_{\Lambda[\cdot]}(\cdot)$ is a constant and, hence, the covariance involving it vanishes. Resorting again to (5) we end up with

$$
\begin{aligned}
& \operatorname{cov}(\left.\Sigma_{\Lambda[\cdot]}(Y(s, W)), \Sigma_{1}(Y(s, W))\right) \\
& \quad=\int_{0}^{s} \mathrm{E} A_{\Lambda[\cdot]}(Y(u, W)) \mathrm{d} u+\int_{0}^{s} \operatorname{var}\left(\Sigma_{\Lambda[\cdot]}(Y(u, W))\right) \mathrm{d} u .
\end{aligned}
$$

Putting together (10) with (11) yields the following expression for $\operatorname{var}\left(\Sigma_{1}(Y(t, W))\right)$ :

$$
\begin{aligned}
\operatorname{var}\left(\Sigma_{1}(Y(t, W))\right) \\
=t \Lambda([W])+\int_{0}^{t} \mathrm{E} \Sigma_{\Lambda[\cdot]}(Y(s, W)) \mathrm{d} s \\
\quad+2\left(\int_{0}^{t} \int_{0}^{s} \mathrm{E} A_{\Lambda[\cdot]}(Y(u, W)) \mathrm{d} u \mathrm{~d} s+\int_{0}^{t} \int_{0}^{s} \operatorname{var}\left(\Sigma_{\Lambda[\cdot]}(Y(u, W))\right) \mathrm{d} u \mathrm{~d} s\right) .
\end{aligned}
$$

It remains to find $\operatorname{var}\left(\Sigma_{\Lambda[\cdot]}(Y(t, W))\right)$. To find an expression, apply (3) again with $\phi=\psi=$ $\Lambda[\cdot]$ to obtain, upon taking expectations and using the fact that $A_{\Lambda[\cdot]}$ is a constant, as remarked in (6) above,

$$
\operatorname{var}\left(\Sigma_{\Lambda[\cdot]}(Y(t, W))\right)=\int_{0}^{t} \mathrm{E} A_{(\Lambda[\cdot])^{2}}(Y(s, W)) \mathrm{d} s
$$

However, using (1),

$$
\begin{aligned}
& A_{(\Lambda[\cdot])^{2}}(Y(s, W)) \\
& =\int_{[W]} \int_{[L \cap W]} \int_{[L \cap W]} 1\left[L \cap L_{1} \text { and } L \cap L_{2} \text { are in the same cell of } Y(s, W)\right] \\
& \times \Lambda\left(\mathrm{d} L_{1}\right) \Lambda\left(\mathrm{d} L_{2}\right) \Lambda(\mathrm{d} L),
\end{aligned}
$$


and, hence,

$$
\begin{aligned}
\operatorname{E~} A_{(\Lambda[\cdot])^{2}}(Y(s, W)) & =\int_{[W]} \int_{[L \cap W]} \int_{[L \cap W]} \mathrm{P}\left(L \cap L_{1} \text { and } L \cap L_{2} \text { are in the same cell of } Y(s, W)\right) \\
= & \int_{[W]} \int_{[L \cap W]} \int_{[L \cap W]} \exp \left(-s \Lambda\left(\left[L_{1}\right) \Lambda\left(\mathrm{d} L_{2}\right) \Lambda(\mathrm{d} L)\right.\right.
\end{aligned}
$$

where $L\left(L_{1}, L_{2}\right)$ stands for the segment joining the points $L \cap L_{1}$ and $L \cap L_{2}$, and where the last equality follows by property 4 of Subsection 2.1 .

To neatly formulate our theory, denote by $\langle\langle(\Lambda \times \Lambda) \cap \Lambda\rangle\rangle$ the segment-intersection measure on the space $\left[\mathbb{R}^{2}\right]$ of finite linear segments in $\mathbb{R}^{2}$ given by

$$
\langle\langle(\Lambda \times \Lambda) \cap \Lambda\rangle\rangle=\int_{\left[\mathbb{R}^{2}\right]} \int_{[L]} \int_{[L]} \delta_{L\left(L_{1}, L_{2}\right)} \Lambda\left(\mathrm{d} L_{1}\right) \Lambda\left(\mathrm{d} L_{2}\right) \Lambda(\mathrm{d} L),
$$

and observe that this defines a locally finite measure, charging finite mass on collections $\overline{[} A \overline{]}$ of segments with both ends falling into a bounded set $A \subset \mathbb{R}^{2}$, because of the local finiteness of $\Lambda$. With this notation, combining (6), (8), (11), (12), (13), and (14) yields the following result.

Theorem 1. For general locally finite and diffuse driving measures $\Lambda$, denoting by

$$
T_{n}^{\exp }(u)=\sum_{k=n}^{\infty} \frac{u^{k}}{k !}=\exp (u)-\sum_{k=0}^{n-1} \frac{u^{k}}{k !}
$$

the nth tail of the exponential series at $u$, we have

$$
\begin{aligned}
\operatorname{var}\left(\Sigma_{\Lambda[\cdot]}(Y(t, W))\right)= & -\int_{\overline{[} W \overline{]}} \frac{T_{1}^{\exp }(-t \Lambda([e]))}{\Lambda([e])}\langle\langle(\Lambda \times \Lambda) \cap \Lambda\rangle\rangle(\mathrm{d} e), \\
\operatorname{cov}\left(\Sigma_{\Lambda[\cdot]}(Y(t, W)), \Sigma_{1}(Y(t, W))\right)= & t\langle\langle\Lambda \cap \Lambda\rangle\rangle(W) \\
& +\int_{\overline{[} W \bar{j}} \frac{T_{2}^{\exp }(-t \Lambda([e]))}{\Lambda([e])^{2}}\langle\langle(\Lambda \times \Lambda) \cap \Lambda\rangle\rangle(\mathrm{d} e),
\end{aligned}
$$

and

$$
\begin{aligned}
\operatorname{var}\left(\Sigma_{1}(Y(t, W))\right)= & t \Lambda([W])+\frac{3 t^{2}}{2}\langle\langle\Lambda \cap \Lambda\rangle\rangle(W) \\
& -2 \int_{[W]} \frac{T_{3}^{\exp }(-t \Lambda([e]))}{\Lambda([e])^{3}}\langle\langle(\Lambda \times \Lambda) \cap \Lambda\rangle\rangle(\mathrm{d} e) .
\end{aligned}
$$

\subsection{Vertex pair correlations for the general case}

In this subsection we also stay in the general setup of locally finite and diffuse $\Lambda$. We will extend the calculations made in Subsection 3.1 to determine the pair-correlation structure of the vertex point process $\mathcal{V}_{Y(t, W)}$ generated by $Y(t, W)$. For definiteness, we adopt the convention that $\mathcal{V}_{Y(t, W)}$ does not include the boundary vertices; this way each vertex arises 
at the intersection of exactly two maximal edges. Recalling the consistency relation $Y(t, W)=$ $Y(t) \cap W$, we see that the covariance structure between bounded regions $U, V \subset \mathbb{R}^{2}$ does not depend on $W$ as soon as both $U$ and $V$ are contained in the interior of $W$. To put this in formal terms, consider the whole-plane covariance measure $\operatorname{cov}\left(\mathcal{V}_{Y(t)}\right)$ of the point process $\mathcal{V}_{Y(t)}$ on $\left(\mathbb{R}^{2}\right)^{2}=\mathbb{R}^{2} \times \mathbb{R}^{2}$ (also called the second-order cumulant measure) given by the relation

$$
\int_{\left(\mathbb{R}^{2}\right)^{2}}(f \otimes g) \mathrm{d} \operatorname{cov}\left(\mathcal{V}_{Y(t)}\right)=\operatorname{cov}\left(\Sigma_{\eta^{f}}(Y(t)), \Sigma_{\eta^{g}}(Y(t))\right),
$$

holding for all bounded measurable $f, g: \mathbb{R}^{2} \rightarrow \mathbb{R}$ with bounded support. Here $\eta^{f}$ is the edge functional

$$
\eta^{f}(e)=\sum_{x \in \operatorname{Vertices}(e)} f(x),
$$

and likewise for $\eta^{g}$. Note that, even though we are apparently dealing with the functionals $\Sigma_{\eta^{f}}(Y(t))$ and $\Sigma_{\eta^{g}}(Y(t))$ defined on the whole-plane process, they can be safely replaced by $\Sigma_{\eta^{f}}(Y(t, W))$ and $\Sigma_{\eta^{g}}(Y(t, W))$, respectively, for some $W$ containing the supports of $f$ and $g$; hence, our martingale relations given in Proposition 1 hold here with no extra assumptions. For the same reasons, all the integrals below with apparently unbounded integration domains are effectively bounded due to the bounded supports of $f$ and $g$, which we are going to exploit without further mention. It is readily seen from (1) that, for each possible realization $Y$ of $Y(t)$ or $Y(t, W)$ in a domain $W$ containing the supports of $f$,

$$
A_{\eta f}(Y)=2 \int_{\left[\mathbb{R}^{2}\right]} \sum_{x \in L \cap Y} f(x) \Lambda(\mathrm{d} L)=2 \Sigma_{\Lambda}{ }_{[\cdot]}(Y),
$$

where $\Lambda^{f}[e]=\int_{[e]} f(e \cap L) \Lambda(\mathrm{d} L)$ and the factor 2 comes from the fact that each point of the tessellation is contained in exactly two maximal edges. Consequently, using (3) with $\phi=\eta^{f}$ and $\psi=\eta^{g}$, and taking expectations, we obtain

$$
\begin{aligned}
\operatorname{cov}\left(\Sigma_{\eta^{f}}(Y(t)), \Sigma_{\eta^{g}}(Y(t))\right)= & \int_{0}^{t} \mathrm{E} A_{\eta^{f} \eta^{g}}(Y(s)) \mathrm{d} s \\
& +2 \int_{0}^{t} \operatorname{cov}\left(\Sigma_{\Lambda^{f}[\cdot]}(Y(s)), \Sigma_{\eta^{g}}(Y(s))\right) \mathrm{d} s \\
& +2 \int_{0}^{t} \operatorname{cov}\left(\Sigma_{\Lambda^{g}[\cdot]}(Y(s)), \Sigma_{\eta^{f}}(Y(s))\right) \mathrm{d} s .
\end{aligned}
$$

Proceeding as in Subsection 3.1, we now turn to the calculation of the $\operatorname{covariance} \operatorname{cov}\left(\Sigma_{\Lambda^{f}}[\cdot]\right.$ $\left.(Y(s)), \Sigma_{\eta^{g}}(Y(s))\right)$. To this end, we note that

$$
A_{\Lambda^{f}[\cdot]}(Y)=\int_{\left[\mathbb{R}^{2}\right]} \Lambda^{f}[L] \Lambda(\mathrm{d} L)=\int_{\mathbb{R}^{2}} f \mathrm{~d}\langle\langle\Lambda \cap \Lambda\rangle\rangle,
$$

whence $A_{\Lambda^{f}[\cdot]}(\cdot)$ is a constant, and we again use (3) with $\phi=\Lambda^{f}$ and $\psi=\eta^{g}$ to obtain, in view of (18),

$$
\begin{aligned}
& \operatorname{cov}\left(\Sigma_{\Lambda^{f}[\cdot]}(Y(s)), \Sigma_{\eta^{g}}(Y(s))\right) \\
& \quad=\int_{0}^{s} \mathrm{E} A_{\Lambda^{f}[\cdot] \eta^{g}}(Y(u)) \mathrm{d} u+2 \int_{0}^{s} \operatorname{cov}\left(\Sigma_{\Lambda^{f}[\cdot]}(Y(u)), \Sigma_{\Lambda^{g}[\cdot]}(Y(u))\right) \mathrm{d} u .
\end{aligned}
$$


Finally, using (3) with $\phi=\Lambda^{f}$ and $\psi=\Lambda^{g}$, and applying (20) yields

$$
\operatorname{cov}\left(\Sigma_{\Lambda^{f}[\cdot]}(Y(u)), \Sigma_{\Lambda^{g}[\cdot]}(Y(u))\right)=\int_{0}^{u} \mathrm{E}_{\Lambda^{f}[\cdot] \Lambda^{g}[\cdot]}(Y(v)) \mathrm{d} v
$$

Thus, using (19), (21) twice, once with $f$ and $g$ interchanged, and then (22), we obtain

$$
\begin{aligned}
\operatorname{cov}\left(\Sigma_{\eta^{f}}(Y(t)), \Sigma_{\eta^{g}}(Y(t))\right) & \\
= & \int_{0}^{t} \mathrm{E} A_{\eta^{f} \eta^{g}}(Y(s)) \mathrm{d} s+2 \int_{0}^{t} \int_{0}^{s} \mathrm{E} A_{\Lambda^{f}[\cdot] \eta^{g}+\Lambda^{g}[\cdot] \eta^{f}}(Y(u)) \mathrm{d} u \mathrm{~d} s \\
& +8 \int_{0}^{t} \int_{0}^{s} \int_{0}^{u} \mathrm{E} A_{\Lambda^{f}[\cdot] \Lambda^{g}[\cdot]}(Y(v)) \mathrm{d} v \mathrm{~d} u \mathrm{~d} s,
\end{aligned}
$$

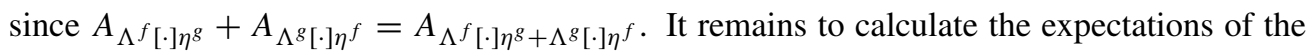
$A_{(\cdot)}$ functionals present in these integrals. However, this is easily done by recalling that, for $L \in\left[\mathbb{R}^{2}\right]$, the intersection $Y(t) \cap L$ is the Poisson point process with intensity measure $L \supseteq A \mapsto t \Lambda([A])$ (see property 4 of Subsection 2.1), whence, in view of (1),

$$
\begin{aligned}
\mathrm{E} A_{\phi}(Y(t)) & =\frac{1}{2} \int_{\left[\mathbb{R}^{2} \overline{]}\right.} \phi(e) \exp (-t \Lambda([e]))\langle\langle(t \Lambda \times t \Lambda) \cap \Lambda\rangle\rangle(\mathrm{d} e) \\
& =\frac{t^{2}}{2} \int_{\overline{[R}^{2} \overline{]}} \phi(e) \exp (-t \Lambda([e]))\langle\langle(\Lambda \times \Lambda) \cap \Lambda\rangle\rangle(\mathrm{d} e)
\end{aligned}
$$

for bounded measurable $\phi$ such that $\phi(\varnothing)=0$, which are locally defined in the sense that there exists a bounded convex $W$ such that $\phi(e)=\phi(e \cap W)$ for all $e$. Note that the extra prefactor of $\frac{1}{2}$ comes from the fact that the segment-intersection measure defined by (15) counts each segment twice, once for each of the two orderings of its two termini. Putting (24) together with (23) yields

$$
\begin{aligned}
\operatorname{cov}\left(\Sigma_{\eta^{f}}(Y(t)), \Sigma_{\eta^{g}}(Y(t))\right) \\
=\frac{1}{2} \int_{\mathbb{R}^{2} \overline{]}} \eta^{f}(e) \eta^{g}(e) I^{1}\left(s^{2} \exp (-t \Lambda([e])) ; t\right)\langle\langle(\Lambda \times \Lambda) \cap \Lambda\rangle\rangle(\mathrm{d} e) \\
\quad+\int_{\left.\overline{[}^{2}\right]}\left(\Lambda^{f}[e] \eta^{g}[e]+\Lambda^{g}[e] \eta^{f}(e)\right) I^{2}\left(s^{2} \exp (-s \Lambda([e])) ; t\right)\langle\langle(\Lambda \times \Lambda) \cap \Lambda\rangle\rangle(\mathrm{d} e) \\
\quad+4 \int_{\overline{[R}^{2} \overline{]}} \Lambda^{f}[e] \Lambda^{g}[e] \mathcal{I}^{3}\left(s^{2} \exp (-s \Lambda([e])) ; t\right)\langle\langle(\Lambda \times \Lambda) \cap \Lambda\rangle\rangle(\mathrm{d} e),
\end{aligned}
$$

where the multiple integral $\mathcal{I}^{n}$ is given by

$$
I^{n}(f(s) ; t):=\int_{0}^{t} \int_{0}^{s_{1}} \cdots \int_{0}^{s_{n-1}} f(s) \mathrm{d} s \mathrm{~d} s_{n-1} \cdots \mathrm{d} s_{1}=\frac{1}{(n-1) !} \int_{0}^{t}(t-s)^{n-1} f(s) \mathrm{d} s,
$$

so that, in particular,

$$
\begin{aligned}
& I^{1}\left(s^{2} \exp (-\lambda s) ; t\right)=\lambda^{-3}\left(2-\left(\lambda^{2} t^{2}+2 \lambda t+2\right) \exp (-\lambda t)\right), \\
& \tau^{2}\left(s^{2} \exp (-\lambda s) ; t\right)=\lambda^{-4}\left(2 \lambda t-6+\left(\lambda^{2} t^{2}+4 \lambda t+6\right) \exp (-\lambda t)\right), \\
& \tau^{3}\left(s^{2} \exp (-\lambda s) ; t\right)=\lambda^{-5}\left(\lambda^{2} t^{2}-6 \lambda t+12-\left(\lambda^{2} t^{2}+6 \lambda t+12\right) \exp (-\lambda t)\right) .
\end{aligned}
$$


For a segment (edge) $e$, consider the measures $\Delta^{e}$ and $\Lambda[\cdot \cap e]$ on $\mathbb{R}^{2}$ that are defined by

$$
\Delta^{e}:=\sum_{x \in \operatorname{Vertices}(e)} \delta_{x}
$$

and

$$
(\Lambda[\cap e])(A)=\Lambda([A \cap e]), \quad A \subseteq \mathbb{R}^{2} .
$$

With this notation, putting together (17) and (25) yields, in view of the definitions of $\eta^{f}$ and $\Lambda^{f}[\cdot]$, the following result.

Theorem 2. For general locally finite and diffuse driving measures $\Lambda$, we have

$$
\begin{aligned}
\operatorname{cov}\left(\mathcal{V}_{Y(t)}\right) & \\
= & \int_{\left.\overline{[}_{\left.\mathbb{R}^{2}\right]}\right]} \frac{1}{2}\left(\Delta^{e} \otimes \Delta^{e}\right) \mathcal{I}^{1}\left(s^{2} \exp (-s \Lambda([e])) ; t\right)\langle\langle(\Lambda \times \Lambda) \cap \Lambda\rangle\rangle(\mathrm{d} e) \\
& +\int_{\overline{\left[\mathbb{R}^{2}\right]}}\left(\Delta^{e} \otimes \Lambda[\cdot \cap e]+\Lambda[\cdot \cap e] \otimes \Delta^{e}\right) I^{2}\left(s^{2} \exp (-s \Lambda([e])) ; t\right)\langle\langle(\Lambda \times \Lambda) \cap \Lambda\rangle\rangle(\mathrm{d} e) \\
& +4 \int_{\left.\overline{[}^{2}\right]}(\Lambda[\cdot \cap e] \otimes \Lambda[\cdot \cap e]) \mathcal{I}^{3}\left(s^{2} \exp (-s \Lambda([e])) ; t\right)\langle\langle(\Lambda \times \Lambda) \cap \Lambda\rangle\rangle(\mathrm{d} e)
\end{aligned}
$$

An intuitive understanding of the structure of the covariance measure in Theorem 2 comes by noting that the first integral in (29) takes into account pairs of vertices constituting the ends of the same maximal edge, the second integral corresponds to pairs of vertices with the property that one of them is an internal vertex of a maximal edge of which the second point is a terminus, whereas the third integral corresponds to pairs of vertices lying on the same maximal edge but not being its termini. Thus, other pairs of vertices (not lying on the same maximal edge) are not present in the covariance measure; roughly speaking, this is because the maximal edges are the only means of propagating dependencies in iteration infinitely divisible tessellations, an intuition to be made more specific in future work.

\subsection{Edge-vertex correlations in the general case}

In this subsection, still placing ourselves in the general setting of a locally finite and diffuse $\Lambda$, we consider the covariance measure between the vertex point process and edge length process generated by $Y(t)$. To this end, define the (random) edge length measure $\varepsilon_{Y(t)}$ of $Y(t)$ by setting, for bounded Borel $A \subseteq \mathbb{R}^{2}$,

$$
\mathcal{E}_{Y(t)}(A)=\sum_{e \in \operatorname{Max} \operatorname{Edges}(Y(t))} \ell(e \cap A),
$$

where $\ell(\cdot)$ stands for the usual one-dimensional length. The object of our interest is the measure $\operatorname{cov}\left(\mathcal{V}_{Y(t)}, \mathcal{E}_{Y(t)}\right)$ given by

$$
\int_{\left(\mathbb{R}^{2}\right)^{2}}(f \otimes g) \mathrm{d} \operatorname{cov}\left(\mathcal{V}_{Y(t)}, \mathcal{E}_{Y(t)}\right)=\operatorname{cov}\left(\Sigma_{\eta^{f}}(Y(t)), \Sigma_{J^{g}}(Y(t))\right)
$$

for bounded measurable $f, g: \mathbb{R}^{2} \rightarrow \mathbb{R}$ with bounded support, where $J^{g}$ denotes the functional $J^{g}(e)=\int_{e} g(x) \ell(\mathrm{d} x)$. Similarly as in (20) we have

$$
A_{J} g(Y)=\int_{\left[\mathbb{R}^{2}\right]} J^{g}(L) \Lambda(\mathrm{d} L) .
$$


Thus, $A_{J^{g}}$ is constant and, hence, using (3) with $\phi=\eta^{f}$ and $\psi=J^{g}$, taking expectations, and recalling (18), yields

$$
\begin{aligned}
\operatorname{cov}\left(\Sigma_{\eta^{f}}(Y(t)), \Sigma_{J^{g}}(Y(t))\right)= & \int_{0}^{t} \mathrm{E} A_{\eta^{f} J^{g}}(Y(s)) \mathrm{d} s \\
& +2 \int_{0}^{t} \operatorname{cov}\left(\Sigma_{\Lambda^{f}[\cdot]}(Y(s)), \Sigma_{J^{g}}(Y(s))\right) \mathrm{d} s .
\end{aligned}
$$

Using (3) once again, with $\phi=\Lambda^{f}[\cdot]$ and $\psi=J^{g}$, upon taking expectations and recalling (20) and (31), we obtain

$$
\operatorname{cov}\left(\Sigma_{\Lambda^{f}[\cdot]}(Y(s)), \Sigma_{J^{g}}(Y(s))\right)=\int_{0}^{s} \mathrm{E} A_{\Lambda^{f}[\cdot] J^{g}}(Y(u)) \mathrm{d} u .
$$

Substituting into (32) leads to

$$
\operatorname{cov}\left(\Sigma_{\eta^{f}}(Y(t)), \Sigma_{J^{g}}(Y(t))\right)=\int_{0}^{t} \mathrm{E} A_{\eta^{f} J^{g}}(Y(s)) \mathrm{d} s+2 \int_{0}^{t} \int_{0}^{s} \mathrm{E} A_{\Lambda^{f}[\cdot] J^{g}}(Y(u)) \mathrm{d} u \mathrm{~d} s .
$$

Applying (24), we obtain

$$
\begin{aligned}
\operatorname{cov}\left(\Sigma_{\eta^{f}}(Y(t)), \Sigma_{J g}(Y(t))\right) & \\
= & \frac{1}{2} \int_{\overline{[R}^{2} \overline{]}} \eta^{f}(e) J^{g}(e) \mathcal{L}^{1}\left(s^{2} \exp (-s \Lambda([e])) ; t\right)\langle\langle(\Lambda \times \Lambda) \cap \Lambda\rangle\rangle(\mathrm{d} e) \\
& +\int_{\left.\overline{[}^{2}\right]} \Lambda^{f}[e] J^{g}(e) I^{2}\left(s^{2} \exp (-s \Lambda([e])) ; t\right)\langle\langle(\Lambda \times \Lambda) \cap \Lambda\rangle\rangle(\mathrm{d} e) .
\end{aligned}
$$

Consequently, putting (34) together with (30) and defining the measure $(\ell(\cdot \cap e))(A):=\ell(A \cap e)$, $A \subseteq \mathbb{R}^{2}$, we obtain the following result.

Theorem 3. For general locally finite and diffuse driving measures $\Lambda$, we have

$$
\begin{aligned}
\operatorname{cov}\left(\mathcal{V}_{Y(t, W)}, \mathcal{E}_{Y(t, W)}\right)=\int_{\overline{[}_{\left.\mathbb{R}^{2}\right]}}( & \frac{1}{2} \Delta^{e} \otimes \ell(\cdot \cap e) \tau^{1}\left(s^{2} \exp (-s \Lambda([e])) ; t\right) \\
& \left.+\Lambda[\cdot \cap e] \otimes \ell(\cdot \cap e) I^{2}\left(s^{2} \exp (-s \Lambda([e])) ; t\right)\right) \\
& \times\langle\langle(\Lambda \times \Lambda) \cap \Lambda\rangle\rangle(\mathrm{d} e) .
\end{aligned}
$$

Observe that the first term in the integral in (35) takes into account pairs consisting of a vertex constituting the terminus of a maximal edge and the maximal edge itself, whereas the second term corresponds to pairs consisting of a vertex lying in the relative interior of a maximal edge and the maximal edge. In analogy to the case of Theorem 2, vertex-edge pairs where the vertex is not adjacent to the edge bring no contribution to the considered covariance structure.

\subsection{Variance calculation for the stationary and isotropic case}

We now specialize the results obtained in Subsection 3.1 to the stationary and isotropic case, i.e. we consider stationary and isotropic random tessellations in the plane that are stable under iteration (STIT tessellations). Up to reparametrization, this means taking the driving measure $\Lambda_{\text {iso }}$, the isometry invariant measure on the space of lines in the plane with length 
density 1. Recall first that Crofton's formula [11, Theorem 5.1.1] for $d=2$ and $k=1$ implies that

$$
\Lambda_{\text {iso }}([K])=\frac{2}{\pi} V_{1}(K)=\frac{1}{\pi} P(K),
$$

where $K \subset \mathbb{R}^{2}$ is a planar convex body with first intrinsic volume $V_{1}(K)$ and perimeter length $P(K)$. For a line segment $e \subset \mathbb{R}^{2}$, this is just

$$
\Lambda_{\text {iso }}([e])=\frac{2}{\pi} \ell(e),
$$

where $\ell(e)$ stands for the length of $e$. It follows from (36) that

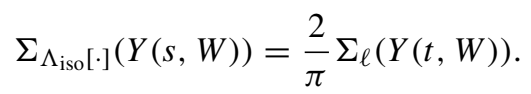

Moreover, in the context of (6) and (7) we have

$$
\left\langle\left\langle\Lambda_{\text {iso }} \cap \Lambda_{\text {iso }}\right\rangle\right\rangle(\mathrm{d} x)=\frac{2}{\pi} \mathrm{d} x \quad \text { and } \quad A_{\Lambda_{\text {iso }[\cdot]}}(Y(s, W))=\frac{2}{\pi} \operatorname{Area}(W) .
$$

Recall from Theorem 4 of [12] that the variance of the total edge length in $W$ of the stationary and isotropic iteration stable random tessellation $Y(u, W), u>0$, simplifies in our particular case to

$$
\operatorname{var}\left(\Sigma_{\ell}(Y(u, W))\right)=\pi \int_{0}^{\infty} \bar{\gamma}_{W}(r)\left(1-\mathrm{e}^{-2 u r / \pi}\right) \frac{\mathrm{d} r}{r},
$$

where $\bar{\gamma}_{W}(r)=\int_{s_{1}} \operatorname{Area}(W \cap(W+r u)) v_{1}(\mathrm{~d} u)$ is the isotropized set-covariance function of the window $W$, with $v_{1}$ standing for the uniform distribution on the unit circle $s_{1}$; see [15] for the definition of $\bar{\gamma}_{W}(\cdot)$ and Subsection 4.2 of [12] for further details. Combining this with (8) and (12), in view of (37) we are immediately led to the variance formula. An alternative method for deriving this formula directly from (16) for the case in which $\Lambda=\Lambda_{\text {iso }}$ is to use (36) and the important identity for the intersection measure (15), namely

$$
\left\langle\left\langle\left(\Lambda_{\text {iso }} \times \Lambda_{\text {iso }}\right) \cap \Lambda_{\text {iso }}\right\rangle(\mathrm{d} \overline{x y})=\frac{4 \mathrm{~d} x \mathrm{~d} y}{\pi^{3}\|x-y\|} .\right.
$$

The above equation may be established using a twofold application of the affine BlaschkePetkantschin formula [11, Theorem 7.2.7], as shown in [12, Equation (50)] in connection with (37). It follows that

$$
\begin{aligned}
\operatorname{var}\left(\Sigma_{1}(Y(t, W))\right) & =\frac{t}{\pi} P(W)+\frac{3}{\pi} \operatorname{Area}(W) t^{2}-2 \int_{W} \int_{W} \frac{T_{3}^{\exp }(-2 t\|x-y\| / \pi)}{(2\|x-y\| / \pi)^{3}} \frac{4 \mathrm{~d} x \mathrm{~d} y}{\pi^{3}\|x-y\|} \\
& =\frac{t}{\pi} P(W)+\frac{3}{\pi} \operatorname{Area}(W) t^{2}-2 \pi \int_{0}^{\infty} \bar{\gamma}_{W}(r) \frac{T_{3}^{\exp }(-2 t r / \pi)}{r^{3}} \mathrm{~d} r .
\end{aligned}
$$

Computing $T_{3}^{\exp }(-2 t r / \pi)$, we arrive at the following result.

Corollary 1. The variance of the number of maximal edges of a stationary and isotropic random iteration stable tessellation $Y(t, W)$ is given by

$$
\begin{aligned}
\operatorname{var}\left(\Sigma_{1}(Y(t, W))\right)= & \frac{t}{\pi} P(W)+\frac{3}{\pi} \operatorname{Area}(W) t^{2} \\
& +\int_{0}^{\infty} \bar{\gamma}_{W}(r)\left(\frac{4 t^{2}}{\pi r}-\frac{4 t}{r^{2}}+\frac{2 \pi}{r^{3}}\left(1-\mathrm{e}^{-2 t r / \pi}\right)\right) \mathrm{d} r .
\end{aligned}
$$


As an example, we may consider for $W$ the ball $B_{R}^{2}$ in $\mathbb{R}^{2}$ with radius $R>0$. In this special case, the isotropized set-covariance function takes the special form

$$
\bar{\gamma}_{B_{R}^{2}}(r)=2 R^{2} \arccos \left(\frac{r}{2 R}\right)-\frac{r}{2} \sqrt{4 R^{2}-r^{2}}, \quad 0 \leq r \leq 2 R .
$$

Unfortunately, the arising integral cannot be further simplified.

We are now interested in the variance asymptotics for a sequence $W_{R}=R W$ of growing observation windows, as $R \rightarrow \infty$. To this end, first note that, asymptotically, as $R \rightarrow \infty$, we have

$$
\int_{0}^{B(R)}\left(\frac{4 t^{2}}{\pi r}-\frac{4 t}{r^{2}}+\frac{2 \pi}{r^{3}}\left(1-\mathrm{e}^{-2 t r / \pi}\right)\right) \mathrm{d} r \sim \frac{4}{\pi} t^{2} \log R,
$$

as long as $\log B(R) \sim \log R$, where $B(R)$ stands for some upper integration bound depending on $R$. Here and later, we will write $f(R) \sim g(R)$ whenever $\lim _{R \rightarrow \infty} f(R) / g(R)=1$. Now, the relation

$$
\bar{\gamma}_{W_{R}} \sim \operatorname{Area}\left(W_{R}\right)=R^{2} \operatorname{Area}(W),
$$

valid uniformly for the argument $r=O(R / \log R)$ and $\bar{\gamma}_{W_{R}} \rightarrow 0$ for $r=\Omega(R \log R)$ (using the standard Landau notation), implies that

$$
\begin{aligned}
\operatorname{var}\left(\Sigma_{1}\left(Y\left(t, W_{R}\right)\right)\right) & \sim \frac{1}{\pi} t P\left(W_{R}\right)+\frac{3}{\pi} t^{2} \operatorname{Area}\left(W_{R}\right)+\frac{4}{\pi} t^{2} \operatorname{Area}\left(W_{R}\right) \log R \\
& \sim \frac{4}{\pi} t^{2} R^{2} \operatorname{Area}(W) \log R .
\end{aligned}
$$

Summarizing, we have shown the following result.

Corollary 2. Asymptotically, as $R \rightarrow \infty$, we have

$$
\operatorname{var}\left(\Sigma_{1}\left(Y\left(t, W_{R}\right)\right)\right) \sim \frac{4}{\pi} t^{2} R^{2} \operatorname{Area}(W) \log R
$$

and

$$
\operatorname{var}\left(N_{v}\left(Y\left(t, W_{R}\right)\right)\right) \sim \frac{16}{\pi} t^{2} R^{2} \operatorname{Area}(W) \log R,
$$

where $N_{v}\left(Y\left(t, W_{R}\right)\right) \sim 2 \Sigma_{1}\left(Y\left(t, W_{R}\right)\right)$ is the number of vertices of $Y\left(t, W_{R}\right)$.

The formulae show that the geometry of the window $W$ is reflected only by its area in the variance asymptotics. Recall from [4] that, for the Poisson-Voronoi tessellation, $\operatorname{PVT}(t, W)$, restricted to some window $W \subset \mathbb{R}^{2}$ with edge length density $t>0$ (i.e. the intensity of the underlying Poisson point process equals $\left.t^{2} / 4\right)$, we asymptotically have

$$
\operatorname{var}\left(N_{v}(\operatorname{PVT}(t, W))\right) \sim 2 t^{2} R^{2} \operatorname{Area}(W) .
$$

Moreover, for the stationary and isotropic Poisson line tessellation, $\operatorname{PLT}(t, W)$, with length intensity $t$ and restricted to $W$, we have, according to [3],

$$
\operatorname{var}\left(N_{v}(\operatorname{PLT}(t, W))\right) \sim \frac{4}{\pi^{2}} t^{3} R^{3} \operatorname{CPI}(W ; 2),
$$

where $\operatorname{CPI}(W ; 2)$ is the second-order chord-power integral of $W$ in the sense of [11, Chapter 8.6]. The appearance of $\mathrm{CPI}(W ; 2)$ in the latter formula means that, beside the area of $W$, its shape also plays an asymptotically important role. The variances of the different tessellation models are illustrated in Figure 2 for a sequence $W_{R}=B_{R}^{2}$ of circles with radii $R$. In this case it holds that $\operatorname{CPI}\left(B_{1}^{2} ; 2\right)=16 \pi / 3$, as can be concluded from Theorem 8.6.6 of [11] with a corrected constant. 


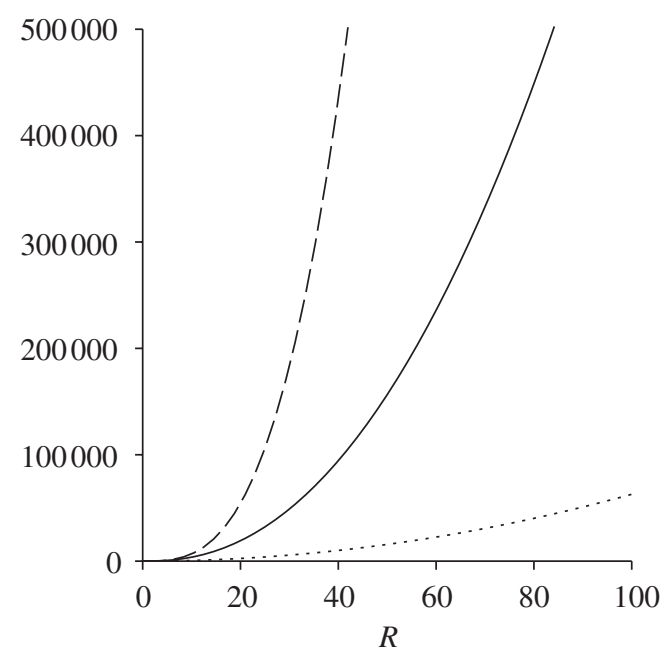

FIgURE 2: Variance comparison for the numbers of vertices of Poisson line (dashed line), STIT (solid line), and Poisson-Voronoi (dotted line) tessellations for $t=1$ and $W_{R}=B_{R}^{2}$, the ball with radius $R>0$.

In particular, the formulae of Corollary 2 establish weak long-range dependencies (cf. [10, p. 60]) for the point process of maximal edge midpoints and the point process of vertices, since

$$
\lim _{R \rightarrow \infty} \frac{\operatorname{var}\left(\Sigma_{1}\left(Y\left(t, W_{R}\right)\right)\right)}{\operatorname{Area}\left(W_{R}\right)}=\lim _{R \rightarrow \infty} \frac{\operatorname{var}\left(N_{v}\left(Y\left(t, W_{R}\right)\right)\right)}{\operatorname{Area}\left(W_{R}\right)}=\infty
$$

As explained at the end of Subsection 3.2, these dependencies are propagated by long maximal edges on which the vertices are lying and the log term in the asymptotic variance formula reflects the weakness of these long-range dependencies. In contrast to the STIT model, Poisson line tessellations have strong long-range dependencies, since $\operatorname{Area}\left(W_{R}\right)^{-1} \operatorname{var}\left(N_{v}\left(\operatorname{PLT}\left(t, W_{R}\right)\right)\right)$ grows polynomially (linearly) in $R$, while Poisson-Voronoi tessellations do not have any longrange dependencies. In fact, the maximal edges almost surely have finite length and there are no full straight lines in the tessellation $Y(t)$. In contrast to this, Poisson line tessellations consist by definition of only full lines and the spatial dependencies are in this case much stronger due to the geometric structure of these processes. For Poisson-Voronoi tessellations, we have independence of local geometries whenever the observation regions are far enough from each other, which means that we 'almost' have independence for the point process of vertices; see [4]. In this sense the STIT tessellations exhibit features intermediate between Poisson-Voronoi and Poisson line tessellations.

\subsection{Vertex pair correlations for the stationary and isotropic case}

Having made very general computations for the covariance measure of the point process of vertices of an iteration infinitely divisible random tessellation $Y(t, W)$ in Subsection 3.2, we now specialize, as in Subsection 3.4, to the stationary and isotropic setup and consider a stationary and isotropic random tessellation $Y(t, W)$ that is iteration stable, i.e. a stationary and isotropic random STIT tessellation with driving measure $\Lambda_{\text {iso }}$. Firstly, recall relation (39). Substituting this expression into (29), using the fact that $\Lambda_{\text {iso }}([\cdot \cap e])=2 \ell(\cdot \cap e) / \pi$ and applying the 
substitution $y=x+u$ yields

$$
\begin{aligned}
& \operatorname{cov}\left(\mathcal{V}_{Y(t)}\right) \\
& =\int_{\mathbb{R}^{2}} \int_{\mathbb{R}^{2}}\left[\frac{1}{2}\left(\Delta^{\overline{O u}} \otimes \Delta^{\overline{O u}}\right) \circ\left(\vartheta_{x}\right)^{-1} \tau^{1}\left(s^{2} \mathrm{e}^{-2\|u\| s / \pi} ; t\right)\right. \\
& +\left(\Delta^{\overline{O u}} \otimes \frac{2}{\pi} \ell(\cdot \cap \overline{O u})+\frac{2}{\pi} \ell(\cdot \cap \overline{O u}) \otimes \Delta^{\overline{O u}}\right) \circ\left(\vartheta_{x}\right)^{-1} \tau^{2}\left(s^{2} \mathrm{e}^{-2\|u\| / \pi s} ; t\right) \\
& \left.+4\left(\frac{2}{\pi} \ell(\cdot \cap \overline{O u}) \otimes \frac{2}{\pi} \ell(\cdot \cap \overline{O u})\right) \circ\left(\vartheta_{x}\right)^{-1} \chi^{3}\left(s^{2} \mathrm{e}^{-2\|u\| s / \pi} ; t\right)\right] \frac{4 \mathrm{~d} x \mathrm{~d} u}{\pi^{3}\|u\|},
\end{aligned}
$$

where we have denoted the origin by $O$ and the usual length measure by $\ell$, and where $\vartheta_{x}$ stands for the diagonal shift $\vartheta_{x}(v, w)=(v+x, w+x), v, w \in \mathbb{R}^{2}$. The covariance measure $\operatorname{cov}\left(\mathcal{V}_{Y(t)}\right)$ can be reduced in the sense of [2, Section 8.1] and, by Proposition 8.1.I(b) therein, the reduced covariance measure $\widehat{\operatorname{cov}}\left(\mathcal{V}_{Y(t)}\right)$ has the form

$$
\begin{aligned}
\widehat{\operatorname{cov}}\left(\mathcal{V}_{Y(t)}\right)=\int_{\mathbb{R}^{2}} & \frac{1}{2}\left(\delta_{u}+\delta_{-u}+2 \delta_{O}\right) \mathcal{I}^{1}\left(s^{2} \mathrm{e}^{-2\|u\| s / \pi} ; t\right) \\
& +\frac{4}{\pi} \ell(\cdot \cap \overline{(-u) u}) \mathcal{I}^{2}\left(s^{2} \mathrm{e}^{-2\|u\| s / \pi} ; t\right) \\
& \left.+4\left(\frac{4}{\pi^{2}} \int_{\overline{O u}} \int_{\overline{O u}} \delta_{v-w} \ell(\mathrm{d} v) \ell(\mathrm{d} w)\right) \mathcal{I}^{3}\left(s^{2} \mathrm{e}^{-2\|u\| s / \pi} ; t\right)\right] \frac{4 \mathrm{~d} u}{\pi^{3}\|u\|},
\end{aligned}
$$

where we have used the facts that

$$
\int \delta_{v-w}\left(\Delta^{\overline{O u}} \otimes \Delta^{\overline{O u}}\right) \mathrm{d}(v, w)=\delta_{u}+\delta_{-u}+2 \delta_{O}
$$

and

$$
\int \delta_{v-w}\left(\Delta^{\overline{O u}} \otimes \ell(\cdot \cap \overline{O u})\right) \mathrm{d}(v, w)=2 \ell(\cdot \cap \overline{(-u) u}) .
$$

Recall now, see [2] again, that the measure $\widehat{\operatorname{cov}}\left(\mathcal{V}_{Y(t)}\right)$ and the reduced second moment measure $\mathcal{K}\left(\mathcal{V}_{Y(t)}\right)$ are related by $\widehat{\operatorname{cov}}\left(\mathcal{V}_{Y(t)}\right)=\mathcal{K}\left(\mathcal{V}_{Y(t)}\right)-\lambda^{2} \ell_{\mathbb{R}^{2}}$ (see [2, Equation (8.1.6)]), where $\ell_{\mathbb{R}^{2}}$ is the Lebesgue measure in the plane and $\lambda$ stands for the intensity of $\mathcal{V}_{Y(t)}$. Hence, taking into account the fact that the vertex intensity $\lambda$ equals $2 t^{2} / \pi$ (see [9]), and transforming into polar coordinates, we obtain

$$
\begin{aligned}
\mathcal{K}\left(\mathcal{V}_{Y(t)}\right)= & \frac{4}{\pi^{3}} \int_{0}^{2 \pi} \int_{0}^{\infty}\left[\left(\delta_{O}+\delta_{\left.r \mathrm{e}^{\mathrm{i} \varphi}\right)} \mathcal{I}^{1}\left(s^{2} \mathrm{e}^{-2 r s / \pi} ; t\right)+\frac{8}{\pi} \ell\left(\cdot \cap \overline{O r \mathrm{e}}{ }^{\mathrm{i} \varphi}\right) I^{2}\left(s^{2} \mathrm{e}^{-2 r s / \pi} ; t\right)\right.\right. \\
& \left.+4\left(\frac{8}{\pi^{2}} \int_{0}^{r}(r-\rho) \delta_{\rho \mathrm{e}^{\mathrm{i} \varphi} \mathrm{d} \rho} \mathrm{d}\right) \mathcal{I}^{3}\left(s^{2} \mathrm{e}^{-2 r s / \pi} ; t\right)\right] \mathrm{d} r \mathrm{~d} \varphi \\
& +\left(\frac{2}{\pi} t^{2}\right)^{2} \ell_{\mathbb{R}}^{2} .
\end{aligned}
$$

From the above expression we can now calculate Ripley's $K$-function,

$$
K(R):=\left(\frac{\pi}{2 t^{2}}\right)^{2} \mathcal{K}\left(B_{R}^{2}\right),
$$

often also considered in the factorial version $\tilde{K}(R)$ with

$$
K(R)=\tilde{K}(R)+\left(\frac{\pi}{2 t^{2}}\right)^{2} \mathcal{K}(\{0\}) ;
$$


see [2, Equation (8.1.12)] or [15, Chapter 4.5]. We obtain

$$
\begin{aligned}
K(R)=\frac{2}{t^{4}} \int_{0}^{\infty}[ & (1+\mathbf{1}[r \leq R]) \mathcal{I}^{1}\left(s^{2} \mathrm{e}^{-2 r s / \pi} ; t\right)+\frac{8}{\pi} \min (r, R) \mathcal{I}^{2}\left(s^{2} \mathrm{e}^{-2 r s / \pi} ; t\right) \\
& \left.+\frac{32}{\pi^{2}}\left(r \min (r, R)-\frac{1}{2} \min (r, R)^{2}\right) \tau^{3}\left(s^{2} \mathrm{e}^{-2 r s / \pi} ; t\right)\right] \mathrm{d} r+\pi R^{2} .
\end{aligned}
$$

Splitting the integral into two parts, one integral over $[0, R]$ and another over $[R, \infty)$, yields

$$
\begin{aligned}
K(R)=\pi R^{2}+\frac{2}{t^{4}} \int_{0}^{R} & {\left[2 \mathcal{I}^{1}\left(s^{2} \mathrm{e}^{-2 r s / \pi} ; t\right)+\frac{8}{\pi} r I^{2}\left(s^{2} \mathrm{e}^{-2 r s / \pi} ; t\right)\right.} \\
& \left.+\frac{16}{\pi^{2}} r^{2} \tau^{3}\left(s^{2} \mathrm{e}^{-2 r s / \pi} ; t\right)\right] \mathrm{d} r \\
+\frac{2}{t^{4}} \int_{R}^{\infty}[ & \tau^{1}\left(s^{2} \mathrm{e}^{-2 r s / \pi} ; t\right)+\frac{8}{\pi} R \tau^{2}\left(s^{2} \mathrm{e}^{-2 r s / \pi} ; t\right) \\
+ & \left.\frac{32}{\pi^{2}}\left(r R-\frac{R^{2}}{2}\right) I^{3}\left(s^{2} \mathrm{e}^{-2 r s / \pi} ; t\right)\right] \mathrm{d} r .
\end{aligned}
$$

Using (26), (27), and (28), we finally obtain Corollary 3 below, using the definition

$$
g(r)=\frac{1}{2 \pi r} \frac{\mathrm{d}}{\mathrm{d} r} K(r)=\frac{1}{2 \pi r} \frac{\mathrm{d}}{\mathrm{d} r} \tilde{K}(r)
$$

for the pair-correlation function, describing the normalized vertex density in terms of the distance $r$ from a typical vertex.

Corollary 3. The pair-correlation function of the vertex point process $\mathcal{V}_{Y(t)}$ of a stationary and isotropic random STIT tessellation $Y(t)$ with edge length density $t>0$ is given by

$$
g(r)=1+\frac{2}{t^{2} r^{2}}-\frac{\pi}{t^{3} r^{3}}+\frac{\pi^{2}}{4 t^{4} r^{4}}-\left(\frac{1}{2 t^{2} r^{2}}-\frac{\pi}{2 t^{3} r^{3}}+\frac{\pi^{2}}{4 t^{4} r^{4}}\right) \mathrm{e}^{-2 t r / \pi} .
$$

Note that in this context in the case of a stationary and isotropic Poisson line tessellation with intensity $t>0$, the pair-correlation function of the point process of vertices takes the form

$$
g^{\operatorname{PLT}(t)}(r)=1+\frac{4}{\pi t r},
$$

which can be easily concluded from Slivnyak's theorem for Poisson point processes; see [15] (here applied to the Poisson process of lines). A comparison between the pair-correlation functions $g(r)$ of the STIT tessellation and $g^{\mathrm{PLT}(t)}(r)$ and that of a Poisson-Voronoi tessellation is shown in Figure 3. However, in contrast to Poisson line and STIT tessellations, the structure of the pair-correlation function of the point process of vertices of a Poisson-Voronoi tessellation is much more complicated. It may be expressed by a sum of integrals of rather involved functions, which cannot be explicitly evaluated. For details and the nontrivial numerical computations, we refer the reader to [4].

It is interesting to compare the pair-correlation formula in Corollary 3 with the information on the variance of the number of edges provided in Corollary 1. To this end, use the variance formula

$$
\operatorname{var}\left(N_{v}(Y(t, W))\right)=2 \pi\left(\frac{2}{\pi} t^{2}\right)^{2} \int_{0}^{\infty} \bar{\gamma}_{W}(r)[g(r)-1] r \mathrm{~d} r+\frac{2}{\pi} t^{2} \operatorname{Area}(W)
$$




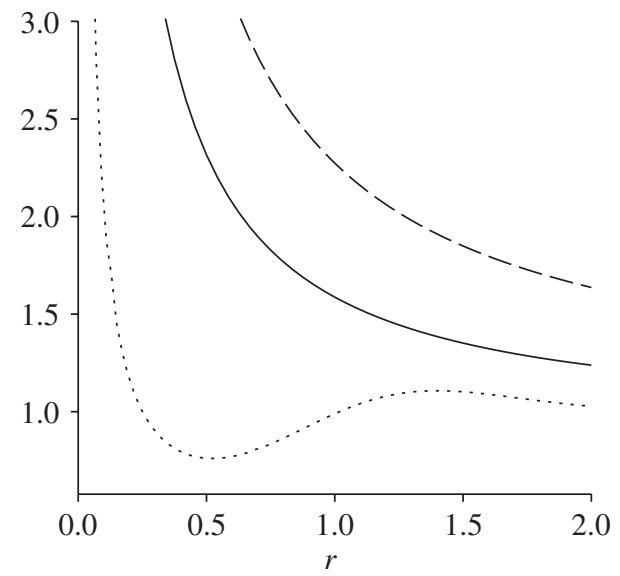

(a)

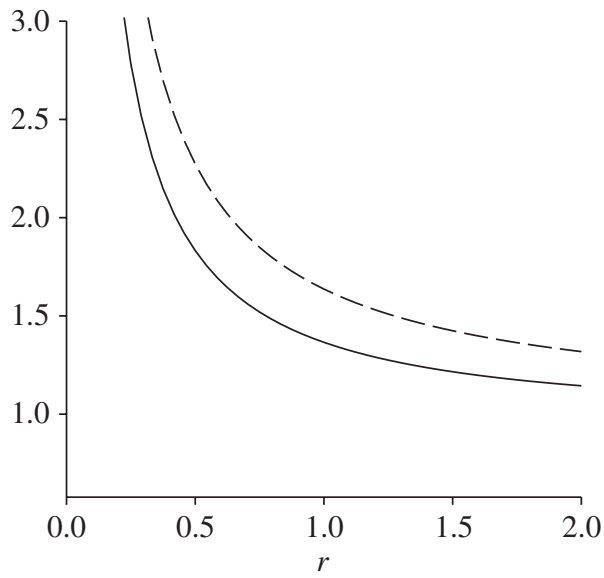

(b)

FIGURE 3: (a) The pair-correlation function of the point process of vertices of Poisson line (dashed line), STIT (solid line), and Poisson-Voronoi tessellations (dotted line). (b) The cross-correlation function of the vertex point process and the length measure of a Poisson line (dashed line) and a STIT (solid line), each time with edge length density 1.

(see [15, Equation (4.5.7)] and Girling's formula thereafter), and compare it with the variance expression in Corollary 1. Taking into account the fact that the number of vertices is equal to, modulo boundary effects, twice the number of edges, we should have agreement between the leading term, $4 t^{2} / \pi r$, in the integral in (40) and $\frac{1}{4} 2 \pi r\left(2 t^{2} / \pi\right)^{2}$ times the leading term, $2 / t^{2} r^{2}$, of $g(r)-1$ in (42), where the factor $\frac{1}{4}$ comes from switching between edge and vertex counts, the factor $2 \pi r$ comes from the transformation into polar coordinates, and the remaining factor is the squared intensity of the vertex point process. Comparing these expressions we readily obtain the required agreement of leading terms, determining the prefactor in the $O\left(R^{2} \log R\right)$-variance asymptotics. The remaining lower-order terms, $o\left(1 / r^{2}\right)$, in the pair correlation function (42) do not have to and do not agree with their suitably normalized counterparts in (40), because the latter contains additional area order corrections and, moreover, takes into account the boundary effects caused by edges hitting the boundary $\partial W_{R}$ of $W_{R}$.

Another aspect that can be compared concerns the radial distribution function. For a stationary and isotropic random point process in the plane with intensity $\lambda>0$ and $K$-function $K(r)$, the radial distribution function $\rho(r)$ is defined by

$$
\rho(r)=\lambda \frac{\mathrm{d} K(r)}{\mathrm{d} r} .
$$

Writing from now on $\rho(r)$ for the radial distribution function of the point process $\mathcal{V}_{Y(t)}$ of vertices of a stationary and isotropic STIT tessellation $Y(t)$ with edge length density $t>0$, we can use (41) to conclude that

$$
\rho(r)=4 t^{2} r+\frac{8}{r}-\frac{4 \pi}{t r^{2}}+\frac{\pi^{2}}{t^{2} r^{3}}-\left(\frac{2}{r}-\frac{2 \pi}{t r^{2}}+\frac{\pi^{2}}{t^{2} r^{3}}\right) \mathrm{e}^{-2 t r / \pi},
$$

whereas, for the radial distribution function $\rho^{\mathrm{PLT}(t)}(r)$ of the vertex point process of a stationary and isotropic Poisson line tessellation with edge length density $t>0$, we obtain

$$
\rho^{\operatorname{PLT}(t)}(r)=2 t^{2} r+\frac{8 t}{\pi}
$$


from Slivnyak's theorem. This means that, asymptotically, we have

$$
\rho(r) \sim 2 \rho^{\mathrm{PLT}(t)}(r) \quad \text { as } r \rightarrow \infty .
$$

Remark 1. There are different normalizations available for the reduced second-moment measure $\mathcal{K}$ and Ripley's $K$-function in the existing literature; see, for example, [2] or [15]. In this paper we decided not to normalize $\mathcal{K}$ by 1 over the squared intensity, $1 / \lambda^{2}$, but we normalize the $K$-function by that factor in order to ensure that the pair-correlation function $g(r)$ tends to 1 as $r \rightarrow \infty$. This is done to keep the formulae consistent with those from previous papers on STIT tessellations. This convention will also be adopted in the next subsection.

\subsection{Edge-vertex correlations in the stationary and isotropic case}

Our interest here is focused on the cross-covariance measure $\operatorname{cov}\left(\mathcal{V}_{Y(t)}, \mathcal{E}_{Y(t)}\right)$ of a stationary and isotropic random STIT tessellation $Y(t)$ with edge length density $t>0$ and driving measure $\Lambda_{\text {iso }}$. It describes the correlations between the stationary and isotropic random point process of vertices and the stationary and isotropic random length measure concentrated on the edges of $Y(t)$. The study of this measure was proposed in [13] and [14], and we recall some general definitions now. Let $\Phi_{1}$ and $\Phi_{2}$ be stationary and isotropic random measures in $\mathbb{R}^{2}$ with respective intensities $\lambda_{1}>0$ and $\lambda_{2}>0$. For a Borel set $B \subset \mathbb{R}^{2}$, we introduce the random measures

$$
\mathcal{K}_{12}(B):=\mathrm{E} \int_{[0,1]^{2}} \Phi_{2}(B+x) \Phi_{1}(\mathrm{~d} x), \quad \mathcal{K}_{21}(B):=\mathrm{E} \int_{[0,1]^{2}} \Phi_{1}(B+x) \Phi_{2}(\mathrm{~d} x) .
$$

The measure $\mathcal{K}_{12}$ describes $\Phi_{2}$ as seen from the typical point of $\Phi_{1}$ and $\mathcal{K}_{21}$ describes the measure $\Phi_{1}$ as seen from the typical point of $\Phi_{2}$ in the sense of Palm distributions. In [14] it was shown that $\mathcal{K}_{12}(B)=\mathcal{K}_{21}(-B)$, which, in particular, implies that, for the ball $B_{r}^{2}$ with radius $r>0$, the identity $\mathcal{K}_{12}\left(B_{r}^{2}\right)=\mathcal{K}_{21}\left(B_{r}^{2}\right)$ holds. The cross $K$-function $K_{12}(r)=K_{21}(r)$ may now be introduced as

$$
K_{12}(r):=\frac{1}{\lambda_{1} \lambda_{2}} \mathcal{K}_{12}\left(B_{r}^{2}\right)
$$

and the cross-correlation function $g_{12}(r)$ of the random measures $\Phi_{1}$ and $\Phi_{2}$ is defined by

$$
g_{12}(r):=\frac{1}{2 \pi r} \frac{\mathrm{d} K_{12}(r)}{\mathrm{d} r}
$$

cf. [13] and [14]. Informally, we could say that $\lambda_{1} K_{21}(r)$ or $\lambda_{2} K_{12}(r)$ is the expectation of $\Phi_{1}\left(B_{r}^{2}+x\right)$ or $\Phi_{2}\left(B_{r}^{2}+x\right)$ at the typical point $x$ of $\Phi_{2}$ or $\Phi_{1}$, respectively. The theory is now applied to our setting and we take for $\Phi_{1}$ the point process $\mathcal{V}_{Y(t)}$ and for $\Phi_{2}$ the random measure $\mathcal{E}_{Y(t)}$ (recall the definitions from Subsection 3.3). We have in this special situation $\lambda_{1}=2 t^{2} / \pi$ and $\lambda_{2}=t$; see [9]. We can now use Theorem 3 together with (36) and (39) to obtain, under the substitution $y=x+u$,

$$
\begin{aligned}
\operatorname{cov}\left(\mathcal{V}_{Y(t)}, \mathcal{E}_{Y(t)}\right)=\int_{\mathbb{R}^{2}} \int_{\mathbb{R}^{2}} & {\left[\frac{1}{2} \Delta^{\overline{O u}} \otimes \ell(\cdot \cap \overline{O u}) \mathcal{I}^{1}\left(s^{2} \mathrm{e}^{-2\|u\| s / \pi} ; t\right)\right.} \\
& \left.+\frac{2}{\pi} \ell(\cdot \cap \overline{O u})^{\otimes 2} \mathcal{I}^{2}\left(s^{2} \mathrm{e}^{-2\|u\| s / \pi} ; t\right)\right] \circ \vartheta_{x}^{-1} \frac{4 \mathrm{~d} u \mathrm{~d} x}{\pi^{3}\|u\|} .
\end{aligned}
$$


Consequently, we end up with

$$
\begin{aligned}
\mathcal{K}_{12}=\int_{\mathbb{R}^{2}} & {\left[\ell(\cdot \cap \overline{O u}) I^{1}\left(s^{2} \mathrm{e}^{-2\|u\| s / \pi} ; t\right)\right.} \\
& \left.+\frac{2}{\pi} \int_{\overline{O u}} \int_{\overline{O u}} \delta_{v-w} \ell(\mathrm{d} v) \ell(\mathrm{d} w) \mathcal{I}^{2}\left(s^{2} \mathrm{e}^{-2\|u\| s / \pi} ; t\right)\right] \frac{4 \mathrm{~d} u \mathrm{~d} x}{\pi^{3}\|u\|}+\frac{2}{\pi} t^{3} \ell_{\mathbb{R}^{2}},
\end{aligned}
$$

whereby, upon passing to polar coordinates, we have

$$
\begin{aligned}
\mathcal{K}_{12}=\frac{4}{\pi^{3}} \int_{0}^{\infty} \int_{0}^{2 \pi} & {\left[\ell\left(\cdot \cap \overline{O r \mathrm{e}^{\mathrm{i} \varphi}}\right) \mathcal{I}^{1}\left(s^{2} \mathrm{e}^{-2 r s / \pi} ; t\right)\right.} \\
& \left.+\frac{4}{\pi}\left(\int_{0}^{r}(r-\rho) \delta_{\rho \mathrm{e}^{\mathrm{i} \varphi}} \mathrm{d} \rho\right) \mathcal{I}^{2}\left(s^{2} \mathrm{e}^{-2 r s / \pi} ; t\right)\right] \mathrm{d} \varphi \mathrm{d} r+\frac{2}{\pi} t^{3} \ell_{\mathbb{R}^{2}} .
\end{aligned}
$$

Recalling the definition of $K_{12}$ and again using the fact that in our setup $\lambda_{1}=2 t^{2} / \pi$ and $\lambda_{2}=t$, we obtain

$$
\begin{aligned}
K_{12}(R)= & \pi R^{2}+\frac{4}{\pi t^{3}} \int_{0}^{\infty}\left[\min (r, R) I^{1}\left(s^{2} \mathrm{e}^{-2 r s / \pi} ; t\right)\right. \\
& \left.+\frac{4}{\pi}\left(r \min (r, R)-\frac{1}{2} \min (r, R)^{2}\right) \mathcal{I}^{2}\left(s^{2} \mathrm{e}^{-2 r s / \pi} ; t\right)\right] \mathrm{d} r \\
= & \pi R^{2}+\frac{4}{\pi t^{3}} \int_{0}^{R}\left[r \mathcal{I}^{1}\left(s^{2} \mathrm{e}^{-2 r s / \pi} ; t\right)+\frac{2}{\pi} r^{2} \tau^{2}\left(s^{2} \mathrm{e}^{-2 r s / \pi} ; t\right)\right] \mathrm{d} r \\
& +\frac{4}{\pi t^{3}} \int_{R}^{\infty}\left[R \mathcal{I}^{1}\left(s^{2} \mathrm{e}^{-2 r s / \pi} ; t\right)+\frac{4}{\pi}\left(r R-\frac{R^{2}}{2}\right) I^{2}\left(s^{2} \mathrm{e}^{-2 r s / \pi} ; t\right)\right] \mathrm{d} r .
\end{aligned}
$$

Using (43) together with (26), (27), and (28), we arrive at the following result.

Corollary 4. The cross-correlation function of the vertex point process $\mathcal{V}_{Y(t)}$ and the random length measure $\mathcal{E}_{Y(t)}$ of a stationary and isotropic random STIT tessellation $Y(t)$ with edge length density $t>0$ is given by

$$
g_{12}(r)=1+\frac{1}{t^{2} r^{2}}-\frac{\pi}{4 t^{3} r^{3}}-\left(\frac{1}{2 t^{2} r^{2}}-\frac{\pi}{4 t^{3} r^{3}}\right) \mathrm{e}^{-2 t r / \pi}
$$

In contrast to this formula, the same cross-correlation function $g_{12}^{\mathrm{PLT}(t)}(r)$ for a stationary and isotropic Poisson line tessellation with edge length density $t>0$ is given by

$$
g_{12}^{\mathrm{PLT}(t)}(r)=1+\frac{2}{\pi t r},
$$

which can be easily obtained from Slivnyak's theorem for which we refer the reader to [15]. A comparison of both functions is shown in Figure 3. We would like to point out that the corresponding cross-correlation function is still unknown for the Poisson-Voronoi model.

\section{Central limit theory}

In this section we will study the functional central limit problem for the total edge count and edge length processes induced by a STIT tessellation in growing windows $W_{R}=R W, R \rightarrow \infty$, 
where $W$ denotes some compact convex set of nonempty interior, to remain fixed throughout the section. We assume that the measure $\Lambda$ is translation invariant, i.e. we are in the STIT regime.

Define the rescaled total edge length process by

$$
\mathcal{L}_{t}^{R, W}:=\frac{1}{R \sqrt{\log R}} \bar{\Sigma}_{\Lambda_{\text {iso }[\cdot]}}\left(Y\left(t+\frac{1}{\log R}, W_{R}\right)\right)
$$

and the rescaled total edge count process by

$$
\mathcal{C}_{t}^{R, W}:=\frac{1}{R \sqrt{\log R}} \bar{\Sigma}_{1}\left(Y\left(t+\frac{1}{\log R}, W_{R}\right)\right), \quad t \in[0,1] .
$$

The main result of this section is the following.

Theorem 4. The processes $\left(\mathcal{L}_{s}^{R, W}, \mathcal{C}_{s}^{R, W}\right)_{s \in[0,1]}$ converge jointly in law, as $R \rightarrow \infty$, on the space $\mathscr{D}\left([0,1] ; \mathbb{R}^{2}\right)$ of $\mathbb{R}^{2}$-valued càdlàg functions on $[0,1]$ endowed with the usual Skorokhod $J_{1}$ topology (see [5, Chapter VI.1] or [1, Chapter 3, Section 14]) to the process $t \mapsto(\xi, t \xi)$, where $\xi$ is a normal random variable with variance $V(\Lambda[\cdot], W)$, which is given by Equation (63) of [12] with $\phi=\Lambda[\cdot]$.

Rather than giving a general formula for $V(\phi, W)$, we refer the reader to Proposition 1 and Equation (72) of [12], where the general case is considered and $V(\phi, W)$ is expressed as a weighted mean width of an associated zonoid and its polar body. Here, we only mention the fact that, for the particular isotropic case, we simply have

$$
V\left(\Lambda_{\text {iso }}[\cdot], W\right)=\frac{4}{\pi} \operatorname{Area}(W) ;
$$

see the discussion following Proposition 1 of [12].

The phenomenon observed in Theorem 4 deserves a short discussion. Namely, although both $\bar{\Sigma}_{\Lambda[\cdot]}\left(Y\left(t, W_{R}\right)\right)$ and $\bar{\Sigma}_{1}\left(Y\left(t, W_{R}\right)\right)$ exhibit fluctuations of the order $R \log R$, the mechanisms in which these fluctuations arise are of a rather different nature.

- As shown in [12, Theorem 6], the leading-order deviations of $\bar{\Sigma}_{\Lambda[\cdot]}$ arise in the initial stages of the MNW construction, usually referred to as the big bang phase. Here, this is the time period $[0,1 / \log R]$. During the later stages of the construction, i.e. the time interval $(1 / \log R, 1]$, the variance increase is of lower order and any newly arising fluctuations are negligible compared to those originating from the big bang. In the asymptotic picture this means the initial fluctuation remains frozen throughout the rest of the dynamics, whence we obtain the constant limit for $\mathcal{L}_{t}^{R}, W$ (note that at this point the Brownian limit for the length process obtained in Theorem 6 of [12] referred to a different time flow).

- In contrast, the deviations of $\bar{\Sigma}_{1}$ arise and cumulate constantly in time $t$ with a rate proportional to $t$ times the initial big bang fluctuation of $\bar{\Sigma}_{\Lambda[\cdot]}$. Thus, as opposed to that of $\bar{\Sigma}_{\Lambda[\cdot]}$, the variance of $\bar{\Sigma}_{1}$ exhibits a nonvanishing quadratic dependency on $t$ even in large- $R$ asymptotics.

Thus, in large- $R$ asymptotics, we have the following intuitive picture. Denoting by $\Xi$ the initial big bang fluctuation of $\Sigma_{\Lambda[\cdot]}$ we can effectively use the following first-order approximations: $\bar{\Sigma}_{\Lambda[\cdot]}\left(Y\left(t, W_{R}\right)\right) \approx \Xi$ and $\bar{\Sigma}_{1}\left(Y\left(t, W_{R}\right)\right) \approx t \Xi$ valid for $t \in(1 / \log R, 1]$. 
Proof of Theorem 4. Consider the auxiliary process

$$
\hat{\Sigma}_{1}(Y(t, W)):=\bar{\Sigma}_{1}(Y(t, W))-\int_{0}^{t} \bar{A}_{1}(Y(s, W)) \mathrm{d} s,
$$

which in view of (5) is the same as

$$
\bar{\Sigma}_{1}(Y(t, W))-\int_{0}^{t} \bar{\Sigma}_{\Lambda[\cdot]}(Y(s, W)) \mathrm{d} s,
$$

and which is a centered $\mathfrak{I}_{t}$-martingale by (2). Squaring and taking expectations, we obtain

$$
\begin{aligned}
\mathrm{E}\left(\hat{\Sigma}_{1}(Y(t, W))\right)^{2}= & \operatorname{var}\left(\Sigma_{1}(Y(t, W))\right)-2 \int_{0}^{t} \mathrm{E}\left[\bar{\Sigma}_{1}(Y(t, W)) \bar{\Sigma}_{\Lambda[\cdot]}(Y(s, W))\right] \mathrm{d} s \\
& +\mathrm{E} \int_{0}^{t} \int_{0}^{t} \bar{\Sigma}_{\Lambda[\cdot]}(Y(s, W)) \bar{\Sigma}_{\Lambda[\cdot]}(Y(u, W)) \mathrm{d} u \mathrm{~d} s .
\end{aligned}
$$

Using the facts that, for $s<t, \mathrm{E}\left[\bar{\Sigma}_{\Lambda[\cdot]}(Y(t, W)) \mid \mathfrak{I}_{S}\right]=\bar{\Sigma}_{\Lambda[\cdot]}(Y(s, W))$ and

$$
\begin{aligned}
\mathrm{E}\left[\bar{\Sigma}_{1}(Y(t, W)) \mid \mathfrak{I}_{s}\right]-\bar{\Sigma}_{1}(Y(s, W)) & =\int_{s}^{t} \mathrm{E}\left[\bar{\Sigma}_{\Lambda[\cdot]}(Y(u, W)) \mid \mathfrak{I}_{s}\right] \mathrm{d} u \\
& =(t-s) \bar{\Sigma}_{\Lambda[\cdot]}(Y(s, W)),
\end{aligned}
$$

as follows by the martingale property of $\bar{\Sigma}_{\Lambda[\cdot]}(Y(t, W))$ and $\hat{\Sigma}_{1}(Y(t, W))$. Taking conditional expectations we can rewrite (44) as

$$
\begin{aligned}
& \mathrm{E}\left(\hat{\Sigma}_{1}(Y(t, W))\right)^{2} \\
& =\operatorname{var}\left(\bar{\Sigma}_{1}(Y(t, W))\right)-2 \int_{0}^{t}(t-s) \mathrm{E} \bar{\Sigma}_{\Lambda[\cdot]}^{2}(Y(s, W)) \mathrm{d} s \\
& \quad-2 \int_{0}^{t} \mathrm{E} \bar{\Sigma}_{1}(Y(s, W)) \bar{\Sigma}_{\Lambda[\cdot]}(Y(s, W)) \mathrm{d} s+2 \int_{0}^{t} \int_{0}^{s} \mathrm{E} \bar{\Sigma}_{\Lambda[\cdot]}^{2}(Y(u, W)) \mathrm{d} u \mathrm{~d} s,
\end{aligned}
$$

and, hence, with the second and fourth terms on the right-hand side canceling out,

$$
\begin{aligned}
\mathrm{E}\left(\hat{\Sigma}_{1}(Y(t, W))\right)^{2}= & \operatorname{var}\left(\Sigma_{1}(Y(t, W))\right) \\
& -2 \int_{0}^{t} \operatorname{cov}\left(\Sigma_{1}(Y(s, W)) \Sigma_{\Lambda[\cdot]}(Y(s, W))\right) \mathrm{d} s .
\end{aligned}
$$

Relation (45) combined with (8) and (10) readily yields

$$
\mathrm{E}\left(\hat{\Sigma}_{1}(Y(t, W))\right)^{2}=t \Lambda([W])+\frac{t^{2}}{2}\langle\langle\Lambda \cap \Lambda\rangle\rangle(W) .
$$

To proceed, define the auxiliary process

$$
\hat{\mathcal{C}}_{t}^{R, W}:=\frac{1}{R \sqrt{\log R}} \hat{\Sigma}_{1}\left(Y\left(t+\frac{1}{\log R}, W_{R}\right)\right)=\mathcal{C}_{t}^{R, W}-\int_{-1 / \log R}^{t} \mathcal{L}_{s}^{R, W} \mathrm{~d} s .
$$

Using the facts that $\Lambda\left(\left[W_{R}\right]\right)=O(R)$ and $\langle\langle\Lambda \cap \Lambda\rangle\rangle\left(W_{R}\right)=O\left(R^{2}\right)$, we conclude from (46) that $\lim _{R \rightarrow 0} \mathrm{E}\left(\hat{\mathcal{C}}_{1}^{R, W}\right)^{2}=0$. Thus, Doob's $L^{2}$-maximal inequality [6, Theorem 3.8(iv)] implies that

$$
\lim _{R \rightarrow \infty} \mathrm{E} \sup _{t \in[0,1]}\left(\hat{\mathcal{C}}_{t}^{R, W}\right)^{2} \leq \lim _{R \rightarrow \infty} 4 \mathrm{E}\left(\hat{\mathcal{C}}_{1}^{R, W}\right)^{2}=0 .
$$


We are now in a position to apply Theorem 6 of [12] (taking into account the fact that the notation $\mathcal{L}^{R, W}$ used in [12] corresponds to a time change of $\mathcal{L}^{R, W}$ as defined here) to conclude that, as $R \rightarrow \infty$,

$$
\left(\mathcal{L}_{t}^{R, W}\right)_{t \in[0,1]} \Rightarrow(\xi)_{t \in[0,1]},
$$

that is, the process $\left(\mathcal{L}_{t}^{R, W}\right)_{t \in[0,1]}$ converges in law in the Skorokhod space $\mathscr{D}([0,1] ; \mathbb{R})$ to the constant process $t \mapsto \xi$, where, recall, $\xi$ is a centered normal random variable with variance $V(\Lambda[\cdot], W)$. Using (48) and recalling definition (47), we see that the processes $\left(\mathcal{L}_{t}^{R, W}, \mathcal{C}_{t}^{R, W}\right)_{t \in[0,1]}$ converge jointly in law in $\mathscr{D}\left([0,1] ; \mathbb{R}^{2}\right)$ to the process

$$
t \mapsto\left(\xi, \int_{0}^{t} \xi \mathrm{d} s\right)=(\xi, t \xi),
$$

which completes the proof of Theorem 4.

Remark 2. For reasons discussed in detail in Remark 6 of [12], we expect the rate of the convergence in Theorem 4 to be rather slow.

\section{Acknowledgements}

The authors would like to thank Joachim Ohser (Darmstadt) for providing the two pictures of simulated STIT tessellations. We are also grateful to the anonymous referees, whose remarks were very helpful in improving the style of this work.

The first author was supported by the Polish Minister of Science and Higher Education, grant number N N201 385234 (2008-2010), and the second author was supported by the Swiss National Science Foundation, grant number PP002-114715/1.

\section{References}

[1] Billingsley, P. (1968). Convergence of Probability Measures. John Wiley, New York.

[2] Daley, D. J. And Vere-Jones, D. (2003). An Introduction to the Theory of Point Processes, Vol. I. Springer, New York.

[3] Heinrich, L. (2009). Central limit theorems for motion-invariant Poisson hyperplanes in expanding convex windows. Rend. Circ. Mat. Palermo 81, 187-212.

[4] Heinrich, L. And Muche, L. (2008). Second-order properties of the point process of nodes in a stationary Voronoi tessellation. Math. Nachr. 281, 350-375.

[5] Jacod, J. ANd Shiryaev, A. N. (2003). Limit Theorems for Stochastic Processes, 2nd edn. Springer, Berlin.

[6] Karatzas, I. And Shreve, S. E. (1998). Brownian Motion and Stochastic Calculus, 2nd edn. Springer, New York.

[7] Mecke, J., Nagel, W. And Weiss, V. (2008). A global construction of homogeneous random planar tessellations that are stable under iteration. Stochastics 80, 51-67.

[8] Nagel, W. AND WeIss, V. (2005). Crack STIT tessellations: characterization of stationary random tessellations stable with respect to iteration. Adv. Appl. Prob. 37, 859-883.

[9] Nagel, W. and Weiss, V. (2006). STIT tessellations in the plane. Rend. Circ. Mat. Palermo 77, 441-458.

[10] SchmidT, H. (2008). Asymptotic Analysis of Stationary Random Tessellations. VDM Verlag, Saarbrücken.

[11] SCHNeIDER, R. AND WeIL, W. (2008). Stochastic and Integral Geometry. Springer, Berlin.

[12] Schreiber, T. AND Thäle, C. (2010). Typical geometry, second-order properties and central limit theory for iteration stable tessellations. Preprint. Available at http://arxiv.org/abs/1001.0990v3.

[13] Stoyan, D. and Ohser, J. (1982). Correlations between planar random structures, with an ecological application. Biometrical J. 24, 631-647.

[14] Stoyan, D. AND OHSER, J. (1985). Cross-correlation measures for weighted random measures and their estimation. Theory Prob. Appl. 29, 345-355.

[15] Stoyan, D., Kendall, W. S. And Mecke, J. (1995). Stochastic Geometry and Its Applications, 2nd edn. John Wiley, Chichester.

[16] Weiss, V., Ohser, J. And Nagel, W. (2010). Second moment measure and $K$-function for planar STIT tessellations. Image Anal. Stereology 29, 121-131. 